\title{
La nourriture des entités surnaturelles dans les croyances populaires néohelléniques
}

Les cas de Kalí ton Oréon et autres génies apportant la prospérité

Alimentation and Supernatural Entities in Modern Greek Folk Belief

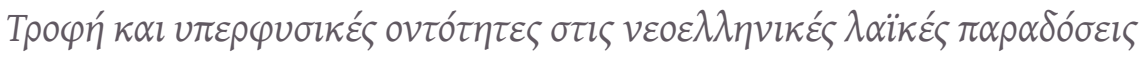

\section{Stamatis Zochios}

\section{(2) OpenEdition}

\section{Journals}

Édition électronique

URL : https://journals.openedition.org/ceb/7158

DOI : $10.4000 /$ ceb.7158

ISSN : 2261-4184

Éditeur

INALCO

Édition imprimée

ISBN : 978-2-85831-230-6

ISSN : 0290-7402

Référence électronique

Stamatis Zochios, «La nourriture des entités surnaturelles dans les croyances populaires néohelléniques », Cahiers balkaniques [En ligne], Hors-série | 2016, mis en ligne le 08 mars 2016, consulté le 06 juillet 2021. URL : http://journals.openedition.org/ceb/7158 ; DOI : https://doi.org/ 10.4000/ceb.7158

Ce document a été généré automatiquement le 6 juillet 2021.

Cahiers balkaniques est mis à disposition selon les termes de la Licence Creative Commons Attribution - Pas d'Utilisation Commerciale 4.0 International. 


\section{La nourriture des entités surnaturelles dans les croyances populaires néohelléniques}

Les cas de Kalí ton Oréon et autres génies apportant la prospérité

Alimentation and Supernatural Entities in Modern Greek Folk Belief

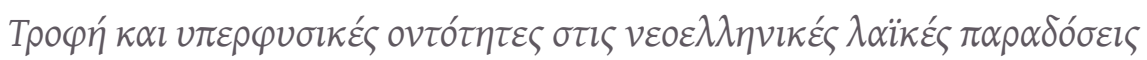

\section{Stamatis Zochios}

\section{La Bonne Dame des montagnes}

1 Le premier tome d'Anecdota Atheniensia du spécialiste de la magie grecque Armand Delatte a été publié en $1927^{1}$. Delatte, depuis 1913, a recueilli dans les Bibliothèques publiques d'Athènes (Bibliothèque Nationale - de la Société historique et ethnographique - du Sénat), un grand nombre de textes inédits. La majorité des manuscrits qui datent $d u \mathrm{XVII}^{\mathrm{e}}$, du XVIII ${ }^{\mathrm{e}}$ siècle et $d u$ XIX ${ }^{\mathrm{e}}$ siècle, sont rédigés en grec moderne et constituent un corpus fondamental pour les domaines de la magie, de la religion et du folklore néohelléniques ${ }^{2}$. Dans un des trente-six textes publiés ici et découvert dans le code bononiensis univers. 3632, écrit au $\mathrm{Xv}^{\mathrm{e}}$ siècle par un médecin nommé Jean d'Aron, se trouve une série de recettes magiques, suivies parfois

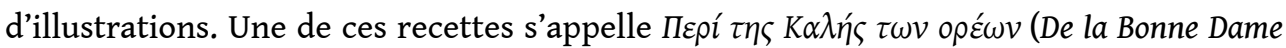
des montagnes ${ }^{3}$ ), et elle est accompagnée d'une illustration (voir page suivante).

2 Comme Delatte l'indique dans l'introduction des recettes, certaines parties du texte sont écrites en signes cryptographiques, les mêmes que ceux utilisés pour les recettes magiques du manuscrit phil.fr. 108,f. 370 de Vienne. Ce système cryptographique apparaît sur les parties gauche et droite de l'illustration ci-dessous, et les deux inscriptions font office de légendes explicatives. À gauche nous lisons : $H \tau \rho \alpha \dot{\pi} \pi \varepsilon \zeta \alpha \tau \tilde{\eta} \varsigma$

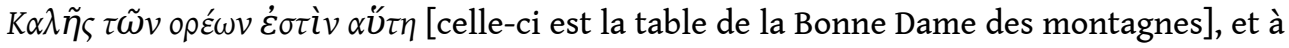

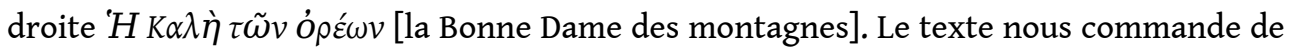


prendre le $1^{\text {er }}$ août une grande assiette avec du miel ${ }^{4}$ et des pommes de pin, et d'aller à midi sur la montagne, poser la nourriture sur une grande pierre et de se cacher en attendant. Ensuite, c'est la Bonne Dame des montagnes - I Kalí ton oréon - qui viendra et demandera : qui a offert tout cela? Et nous, nous répondrons : Moi. Et ensuite elle fera tout ce que nous désirons. Par conséquent, la recette concerne la propitiation d'une figure hybride, mi-femme, mi-serpent de type mélusinien avec une couronne, à savoir avec un statut surhumain, à laquelle une table chargée de nourritures est offerte. Cette dame s'appelle Kalí ton oréon.

\section{La table de la Bonne Dame des montagnes}

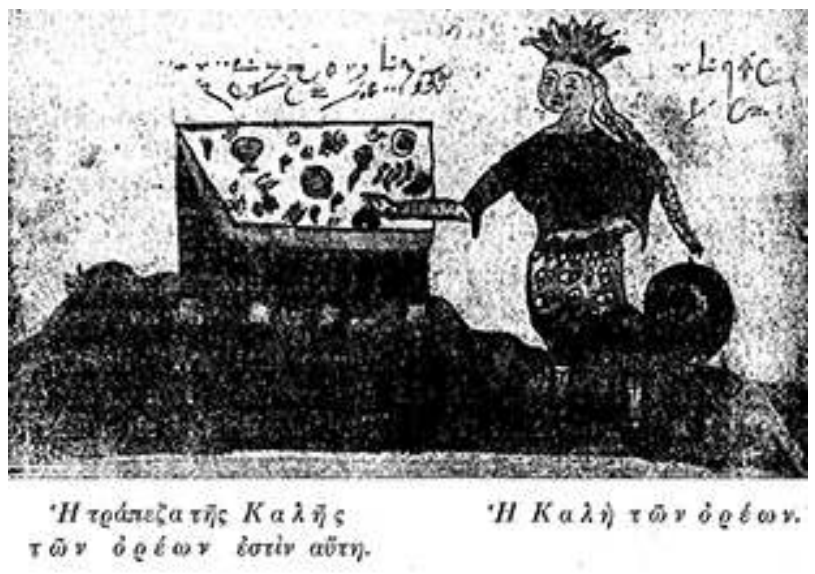

Delatte Armand, Anecdota Atheniensia, T. ı, Liège, Imp. H. Vaillant-Carmanne ; Paris, É. Champion, 1927, p. 600.

Le savant polyvalent $\mathrm{du} \mathrm{XI}^{\mathrm{e}}$ siècle, Michael Psellos, dans son traité relatif au terme connu depuis l'Antiquité ephialtès ${ }^{5}$, considère Kalí ton oréon comme un synonyme du démon baboutzikários, figure obscure du folklore byzantin et du démon barychnás, à savoir de la version byzantine - et par la suite aussi néohellénique - du cauchemar ${ }^{6}$. Cette identification entre la Bonne Dame, le baboutzikários et le barychnás semble complexe. Allatios, le savant gréco-italien du xvII siècle signale à ce sujet que barychnás, baboutzikários ou ephialtès n'ont rien à faire avec la Bonne Dame des bois ou des montagnes (pulcram nemorum sive montium) ${ }^{7}$ et que Psellos lui-même savait bien que la dame des montagnes n'est rien de plus que ce qu'on appelle communément Pulchras Dominas (à savoir néraïdes), qui n'ont rien à voir avec barychnás et ephialtès.

Cette référence pséllienne à Kalí ton oréon a été reprise par Skarlátos Vyzántios dans son

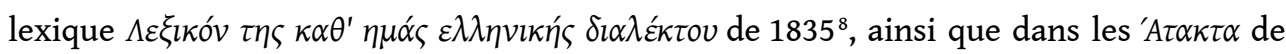
Koraïs. Selon l'érudit grec, ephialtès ou baboutzikários est un démon nocturne qu'on appelle aussi Kalí ton oréon (entre parenthèses il donne la variante ou traduction française, la belle des montagnes), synonyme des Kalés arxóntises (entre parenthèses Koraïs ajoute la version française belles-dames), euphémismes tous les deux pour néraïdes-néréides ${ }^{9}$. Le père du folklore néohellénique Nikólaos Polítis, dans son ouvrage

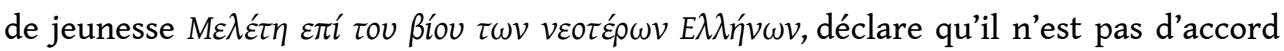
avec Koraïs et cette théorie d'euphémisme ; on attribue cette appellation kali à Kalí ton oréon ou kalí kyría, kalokyría ou kalí arxóntisa parce qu'elle peut être bénéfique, et non pas pour déguiser son aspect désagréable ${ }^{10}$. En effet, les documents justifient cette opinion. Selon la recette magique des Anecdota Atheniansia citée ci-dessous, Kalí ton oréon peut se révéler manifestement bénéfique surtout à travers le motif de la table dressée 
( $\eta \tau \rho \alpha ́ \pi \varepsilon \zeta \alpha)$ : on prépare une table pour l'entité surnaturelle, et si elle est satisfaite de ces offrandes, elle récompense la personne. Il s'agit apparemment d'une pratique rituelle de la III $^{\text {e }}$ fonction selon la fameuse tripartition de Georges Dumézil, cette fonction étant celle de la prospérité ${ }^{11}$.

\section{Les fées de la prospérité}

Dans le monde néohellénique on retrouve le motif des tables dressées dans plusieurs cas, notamment dans les récits de voyage de Ludwig Ross, Dora d'Istria, Edward Dodwell, Jean-Jacques Ampère ou François Pouqueville. L'archéologue allemand Ludwig Ross ayant séjourné en Grèce de 1833 à 1843 pour devenir le premier professeur d'archéologie à l'Université d'Athènes, note entre autres que, dans une grotte à Kiphissia et dans de nombreux autres endroits de Grèce, les gens offrent des galettes de

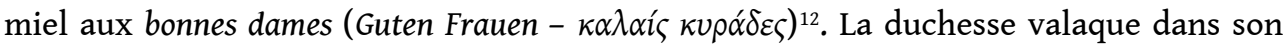
ouvrage sur les femmes de l'Orient et plus précisément dans le chapitre concernant « la femme hellène ", nous informe que la jeune fille " charge sa nourrice ( $\beta \alpha i i ́ \alpha)$ de faire aux mires ( $\mu$ oípal) - c'est le nom des Parques - une offrande de gâteaux et de miel, afin d'avoir un mari selon son cœur $»^{13}$. À son tour, l'archéologue irlandais Dodwell donne sa propre version de la coutume :

While I was drawing the outside of this sepulchral chamber, two Turkish women arriving seemed much disconcerted at my presence; [...] The Greek who was with me said he was certain they had performing magic ceremonies, as the cavern was haunted by the Moipal, or Destinies. I found in the inner chamber a small feast, consisting of a cup of honey and white almonds, a cake, on a little napkin, and a vase of aromatic herbs burning, and exhaling an agreeable perfume. This votive offering was placed upon a rock, which was cut and flat at top, and was probably originally an altar or table, on which an annual sacrificial ceremony was performed by the relations of the deceased ${ }^{14}$.

6 L'hypothèse de Dodwell est correcte. La roche servait de table du même type que celle croisée dans la recette magique de Kalí ton oréon. Il est remarquable que, dans le melting pot de l'Empire ottoman, les femmes turques entrent dans la caverne des Moires pour les vénérer, en nous rappelant que le caractère des croyances populaires n'est pas rigide et n'obéit pas strictement à une catégorisation selon des critères, par exemple nationaux (une tradition grecque peut être retrouvée en Bulgarie, en Albanie ou en Turquie et l'inverse). De plus, comme Dodwell l'explique plus bas, la raison de ce rituel est la fertilité-prospérité : "the offerings they (les femmes) had made to the Destinies, in order to render them propitious to their conjugal speculations" 15 .

7 Par ailleurs, le savant français Jean-Jacques Ampère signale en 1844 que «les Grecs croient aux Parques et les appellent de leur ancien nom Moirai. Trois jours après la naissance d'un enfant on prépare un festin pour elles ${ }^{16}$. Enfin, le médecin et diplomate philhellène Pouqueville mentionne :

[...] qu'on célèbre au cinquième jour de l'accouchement, l'amphidromie qui est maintenant appelée la visite des mires ${ }^{17}$. La plus pauvre cabane prend alors un air de fête pour recevoir les bonnes demoiselles, qu'on ne se voit jamais, quoiqu'elles emportent la fièvre de lait de l'accouchée, $\varepsilon \lambda_{\imath} \kappa \omega ́ v \alpha$ (il veut dire $\left.\lambda \varepsilon \chi \omega ́ v \alpha\right)$. Malgré cette attentive bonté, il faut se garder de la laisser seule, dans la crainte qu'elles ne

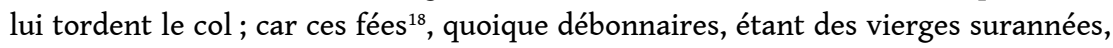
envient aux femmes le bonheur d'être mères ${ }^{19}$. 

l'Antiquité via les visites des mires, est complété par d'autres écrits: dans son cinquième volume, Pouqueville se réfère au grand cachot, " une offrande aux mires, consistant en un gâteau de froment, une coupe remplie de miel et quelques grains d'encens déposés sur une petite nappe en toile neuve de coton $»^{20}$. Ailleurs, il cite en grec une incantation accompagnant les pratiques d'invocation des mires ou des fées:

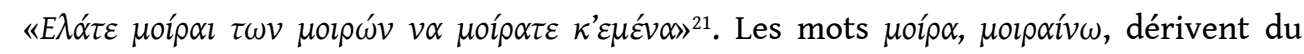
verbe $\mu \varepsilon i ́ p o \mu \alpha l$, «recevoir une partie, un morceau ». Par l'action des mires il devient alors entendu qu'on reçoit une certaine chose, bonne ou mauvaise, de ces femmes. Pour que la chose soit bonne, il fallait s'exercer aux échanges.

Les voyageurs figurent parmi les premiers observateurs du folklore néo-hellénique et des traditions populaires. Les folkloristes, à leur tour, ont fourni des informations similaires. Ainsi, en Béotie, les jeunes filles se rendent aux sources des villages au crépuscule la veille du Nouvel An en tenant un pichet $(\sigma \tau \alpha \dot{\mu} \mu \nu \alpha)$ et une assiette creuse

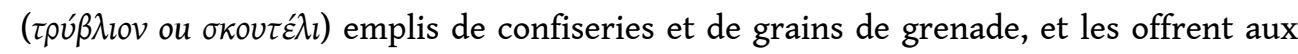
Moires (décrites en tant que jeunes femmes avec les cheveux blonds qui se coiffent avec des peignes dorés juste comme les fées), pour qu'elles leur fassent plaisir ${ }^{22}$. À Syros, pendant les trois premiers soirs après la naissance de l'enfant, on pose sur la table une jarre de gâteau, trois petites cuillères et trois verres d'eau pour les mires qui viendront décider du destin ( $v \alpha \mu o r p \alpha ́ v o v v)$ du nouveau-né ${ }^{23}$.

10 Les offrandes concernant cette procédure de décision varient. En Arcadie, on pose sous l'oreiller du pain, de l'encens, du sucre, et si l'enfant est un garçon, du papier et un porte-plume, si c'est une fille, une anguille ${ }^{24}$. À Kozani sont placés sous le berceau du nouveau-né un pain et trois oignons ${ }^{25}$. À Rhodes sur la table, un plat de miel ${ }^{26}$. À Karpathos, le septième soir après la naissance, on croit que les mires arrivent pour distribuer leurs cadeaux à l'enfant, aussi la sage-femme pose-t-elle sur la table couverte d'une nappe blanche du miel, tout comme du pain blanc, une carafe d'eau, du sel, des oignons blancs, de l'encens ${ }^{27}$. Il semble que le miel et les galettes à base de cette substance qui apparaissent dans presque tous les cas d'offrandes déjà examinés, étaient aussi indispensables au sein des pratiques propitiatoires des dieux antiques, phénomène qui explique les mots de Strepsiade dans les Nuées d'Aristophane : « Metsmoi donc maintenant entre les mains un gâteau miellé. J'ai peur, en entrant là-dedans, comme si je descendais dans l'antre de Trophonios $»^{28}$. Trophonios, héros bénéfiquepossesseur de l'oracle de Béotie, pour devenir favorable au croyant, doit recevoir des gâteaux de miel après la descente du fidèle dans l'abaton et la préparation pour

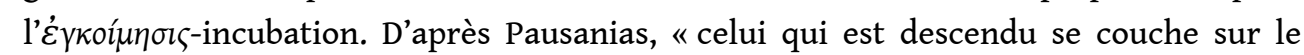
carrelage, et tenant à chaque main un gâteau pétri avec du miel, il met ses pieds dans cette ouverture, et cherche à y entrer jusqu'aux genoux ${ }^{29}$.

11 Le motif de la table des fées et des mires réapparaît dans un autre genre de la littérature populaire grecque : les contes. Dans un tel exemple, un roi et une reine organisent une

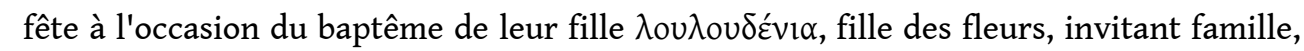
amis et trois (sept ou douze selon d'autres versions) fées marraines. Les deux premières font un don à la princesse : beauté et richesse. Mais la troisième, Movouó́ca (qui a un œil), furieuse de ne pas avoir été invitée lui lance un sort: que louloudénia meure a

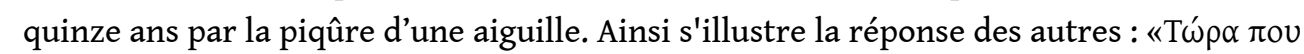




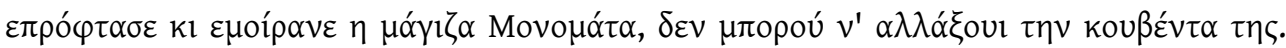

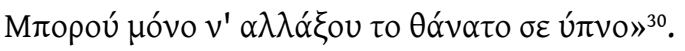

Il est entendu que le conte, ainsi que ses multiples versions ${ }^{31}$, appartiennent au type AT 410 (The sleeping beauty - la Belle au bois dormant) du système des Aarne-Thompson qui nous est connu surtout grâce aux conteurs, Perrault, les frères Grimm et Giambattista Basile. Le type AT 410 dont le motif de la table des fées marraines fait partie, existe pourtant déjà depuis le Moyen Âge: il apparaît dans le Roman de Perceforest (partie III, tome III) du xie siècle avec l'histoire romantique de Troillus et de Zellandine ${ }^{32}$. À la même époque, la coutume a été rapportée comme une superstition par plusieurs théologiens, selon lesquels les femmes décrites n'étaient que démons ou créatures-créations-illusions du diable. Ainsi, souvent évoquée en tant que mensas ornare, la pratique, placée dans le cadre calendaire des douze jours de Noël, période fondamentale dans le folklore européen, dont l'origine préchrétienne, liée au solstice d'hiver, est prouvée, a préoccupé une série d'auteurs cléricaux ${ }^{33}:$ chez les Romains par exemple, dresser une table à cette époque de l'année correspondait à un rite religieux lié au culte des ancêtres, dispensateurs de la fertilité du sol et de la fécondité des hommes et des bêtes.

Ces Douze jours ( $\delta \omega \delta \varepsilon \kappa \alpha \eta ́ \eta \varepsilon \rho 0)$ sont aussi une période significative pour le folklore grec, riche de coutumes et de croyances populaires. En revanche, en Grèce, les néraïdes, les mires et ses tables sont très rarement relatives à cette période et sont plus ou moins absentes du folklore des Douze Jours, en étant « remplacées » par un être surnaturel, exclusivement spécifique identique à cette période, le fameux kalikantzaros.

\section{Le kalikantzaros et les stoicheiá affamés : êtres maléfiques et bénéfiques}

Le kalikantzaros est présenté par les croyances populaires comme un être maléfique avec des traits physiques répulsifs et une action particulièrement menaçante. Il est, entre autres, constamment affamé. Il mange des choses impures (vers, mouches, crapauds, serpents et autres reptiles), et il aspire à entrer dans maisons pour y manger

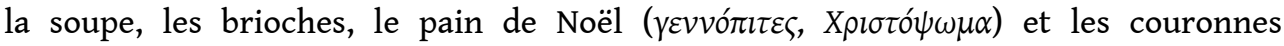

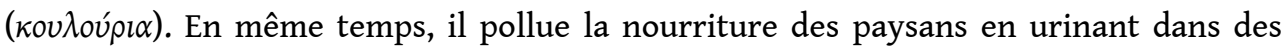
plats, sur les produits alimentaires et surtout sur la farine ${ }^{34}$.

On retrouve ce motif de manger une nourriture impure et interdite, ou de polluer la nourriture humaine, au sein de la mythologie du revenant grec, vrikolakas, qui mâche son linceul, le drap mortuaire, en provoquant des maladies, boit du sang, mange de la chair humaine, et parfois les nourritures des êtres humains, principalement la farine. Manger une nourriture interdite, à savoir chair et sang, polluer ou voler-manger la nourriture des êtres humains, signifie non seulement une suppression de la $\mathrm{III}^{\mathrm{e}}$ fonction dumézilienne, celle de la prospérité, mais aussi un inversement de la prospérité, une contre-prospérité.

Cependant le kalikantzaros n'est pas uniquement un être de contre-prospérité. Il

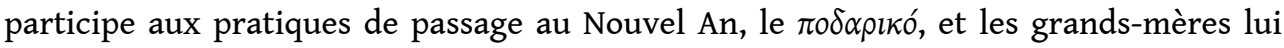
font, au coin du toit, des offrandes de nourriture (une assiette avec des saucisses ou de

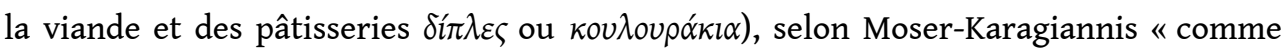
faisaient les Anciens pour la divinité aux trois visages Hécate $»^{35}$. Et elle continue : «À 
propos des offrandes sur la table du repas de fête, qui se pratiquent ici pour les kalikantzaroi, il faut noter qu'elles se pratiquent aussi pour d'autres, et en particulier le Christ, la Vierge et saint Basile. Enfin, quand ils vont partir, le dernier jour du Dodécamère, pour qu'ils soient contents, on les gâte et on leur offre des fritures sucrées... $\aleph^{36}$. Il serait intéressant de noter ici que cette distribution de nourriture, et surtout de pâtisseries, aux kalikantzaroi à la fin des Douze Jours, rappelle la distribution de denrées alimentaires - surtout de gâteaux - aux carnavalistes de la fin de cette période sacrée - les momogeroi, les arkoudes, les rougatsaria, qui, déguisés avec des peaux d'animaux et de masques monstrueux, passent d'une maison à l'autre pour demander des offrandes et distribuer, si ils reçoivent ce qu'ils exigent, la prospérité. Pour Polítis, ces déguisements ont poussé l'imagination du peuple à la création des kalikanzaroi ; les similarités au physique, à l'action et au nom ( une origine commune ${ }^{37}$. Si la théorie de Polítis est valide, les nourritures offertes aux carnavalistes pour s'assurer la prospérité, coïncident avec la nourriture offerte pour la propitiation des kalikantzaroi.

17 Par conséquent, on comprend que le kalikantzaros, comme par ailleurs la plupart des êtres surnaturels, n'est pas purement maléfique mais peut manifester une ambivalence et un dualisme suivant le bipôle aspect bénéfique/prospérité contre aspect maléfique/ contre-prospérité, tout comme les mires et les néraïdes. Pour la propitiation de ces êtres, la pratique principale a été l'offre des nourritures, souvent réalisée par le dressement d'une table.

Par ailleurs de nombreuses traditions grecques nous informent que les femmes préparent le soir de grands pichets, $\sigma \tau \alpha \dot{\mu} \mu v \varepsilon \varsigma$, et diverses nourritures pour que le génie

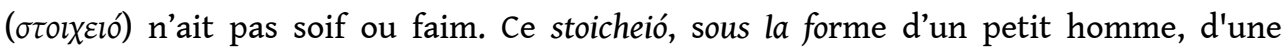
ombre, d'un espèce d'ancêtre mort, d'un lézard et principalement d'un serpent

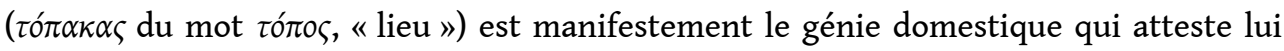
aussi un dualisme caractéristique: si on le traite bien, il considère l'habitant de manière bénéfique en offrant richesse, protection ou fertilité, si on le traite mal, il est maléfique envers l'habitant. Ainsi, il devient un poltergeist ou une cause de pauvreté, en démolissant la maison. C'est pour ces raisons que dans les anciennes maisons rurales il y a avait un coin dédié au génie de la maison. Les résidents disposaient dans cet espace sacré de la nourriture ou d'autres objets offerts au génie ${ }^{38}$.

Selon Allatios, à Chios, le maître de maison entre dans les chambres le jour du Nouvel An avec un panier plein de fruits, de gâteaux et de pains, pour la prospérité de la maison ${ }^{39}$. Enfin, quand on distribue la $\beta \alpha \sigma \imath \lambda o ́ \pi \imath \tau \alpha$, le gâteau du Nouvel An, le premier morceau est pour la maison, à savoir pour le génie de la maison. Il est indispensable de nourrir le génie du lieu au niveau des fondations de la maison pour le rendre favorable. Lorsqu'on pose la première pierre ou la pierre angulaire, il faut offrir des nourritures au génie, voire procéder à un sacrifice, sacrifice d'un animal ou d'une ombre, à interpréter comme un sacrifice humain virtuel. Dans ce cas, sacrifier voulait principalement dire «nourrir ». Le génie se nourrit de sang par cette propitiation qui sacralise le lieu et qui permet par la suite à l'habitant de bâtir la maison. Souvent ce génie est un ancêtre. Par le sacrifice d'un animal, de l'ombre d'un être vivant et le sang versé, le mort se nourrit de vie, et, tranquillisé peut accepter la transformation de l'espace sauvage et profane en espace habité et sacré ${ }^{40}$.

On retrouve le motif du sang versé dans les récits concernant la chasse aux trésors ${ }^{41}$. Ici aussi les mêmes motifs narratologiques reviennent à plusieurs reprises : l'être humain 
rêve d'un saint ou d'un $\alpha \rho \alpha ́ \pi \eta \varsigma$ (homme noir-sarrasin), qui lui indique l'endroit du trésor; l'être humain doit le trouver et procéder méthodiquement au sacrifice nécessaire enfin acquérir les objets de valeur. Une première condition préalable au succès est de rester silencieux. Dans le cas contraire, le trésor se transforme en matière sans valeur, généralement en charbon. Une deuxième condition est d'exterminer le gardien du trésor qui est protégé par un arápis, un géant, un serpent, un ancêtre ou un autre être surnaturel. Une méthode alternative est la propitiation du gardien. La Solomonikí, le plus fameux grimoire néo-hellénique et version grecque de Clavicula Salomonis, explicite la démarche à suivre en donnant des instructions précises: il faut sacrifier un coq, plus précisément un coq noir, ou s'autoblesser, faire couler du sang, et nourrir ainsi le génie qui protège le trésor. En le rendant favorable, on peut ainsi obtenir le trésor.

\section{Les offrandes en tant qu'acte propitiatoire}

Nous avons examiné une série de catégories d'êtres surnaturels (néraïdes, mires, kalikantzaros, génie de la maison, génie du trésor) et un motif mythique, selon lequel on leur offre des nourritures. Le contexte narratologique est différent pour chaque catégorie et les motifs ne sont pas toujours développés dans la même manière : les mires exigent qu'une table soit dressée et préparée, alors que le génie des trésors demande un acte magique-rituel. Par ailleurs, les nourritures ne sont pas identiques : les néraïdes préfèrent le miel et les galettes, le génie du trésor témoigne de goûts plus cruels et alimentairement interdits. Cependant, la conséquence reste similaire: si l'être surnaturel est satisfait des offrandes, il récompense la personne avec des cadeaux matériels ou immatériels. Si les offrandes ne lui suffisent pas, il punit les habitants, d'une façon parfois grave.

D’un côté nous avons un dualisme évident, qui est caractéristique des déités païennes,

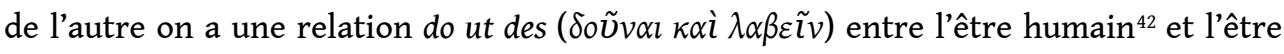
surnaturel. Cette relation do ut des est absente, même interdite, dans le christianisme canonique. C'est une survivance préchrétienne absorbée par la cosmologie néohellénique, qui est certainement orthodoxe, mais qui ne suit pas strictement à la lettre $\mathrm{du}$ dogme religieux. On revient donc au phénomène du christianisme parallèle, christianisme populaire, qui se forme autour du noyau du dogme orthodoxe en n'oubliant pas les origines païennes. Au sein de ce christianisme, un réseau étendu de croyances et coutumes préchrétiennes camouflées a survécu.

Un premier exemple de tradition préchrétienne adaptée au système religieux du christianisme est le gâteau du jour de l'An, la $\beta \alpha \sigma \imath \lambda o ́ m \imath \tau \alpha$ citée ci-dessus, dont le premier morceau est offert à la maison, à savoir au génie domestique, et les suivants à Jésus Christ, Marie et saint Basile.

Un deuxième exemple est une tradition rapportée par Aristídis Tatarákis et selon laquelle à Milos, à la Sainte Catherine, le 25 novembre, les jeunes vierges préparent des galettes ( $\pi \lambda \alpha \kappa o u ́ v \tau \alpha \varsigma)$ salées, les consomment et ensuite se couchent en chantant cette

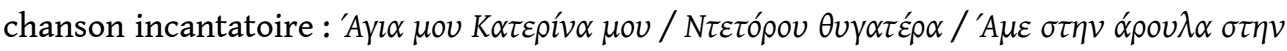

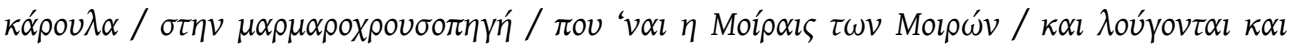

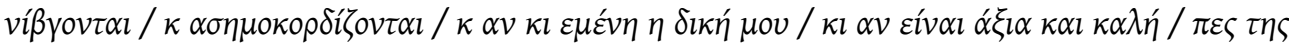
$v \alpha{ }^{\prime} \rho \theta \varepsilon l v \alpha \mu \varepsilon \beta \rho \varepsilon l^{43}$. 


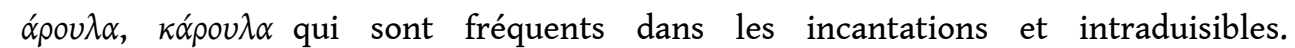

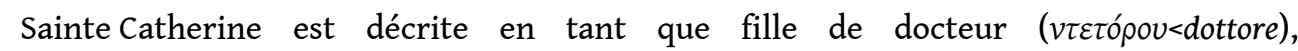
caractéristique étonnante, vu qu'il est connu qu'elle était la fille du roi Costus. Il pourrait pourtant signifier que Sainte Catherine fut instruite dans tous les arts libéraux. La chanson-incantation continue en exhortant la sainte d'aller à la source d'eau construite en or et en marbre, où les moires se baignent et se lavent ${ }^{44}$ pour demander à la mire de la fille de venir la voir (pour qu'elle puisse lui demander ensuite ce qu'elle désire). Il s'agit d'une incantation qui s'inscrit sans doute dans les croyances relatives aux mires. Mais ce qui est frappant dans le texte particulier est que Sainte Catherine, favorisée par les offrandes des galettes, devient un médiateur entre l'être humain et les êtres divins. Ce poème est un exemple extraordinaire du syncrétisme religieux de la Grèce moderne qui s'est formé entre le présent chrétien orthodoxe (Sainte Catherine) et le passée préchrétien (mires).

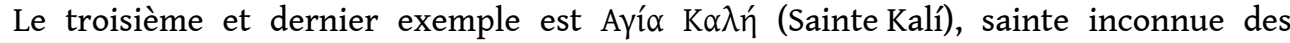
synaxaristes et qui n'est pas fêtée. On la croise principalement dans le poème médiéval

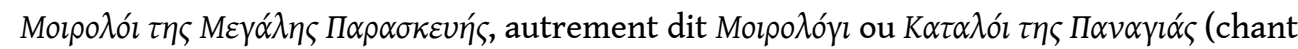
funèbre de Marie la Vierge), ses versions prosaïques et certaines narrations populaires ${ }^{45}$. Dans le Moipoló, Agía Kalí accuse la Vierge Marie de s'être mise à table à la suite de la crucifixion, pour le repas traditionnel du souper des funérailles ( $\mu \alpha \kappa \alpha \rho i \alpha ́$,

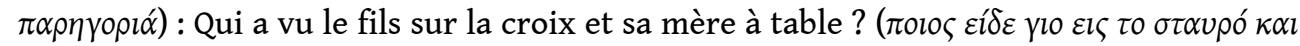

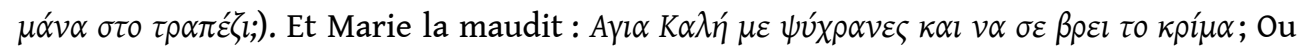

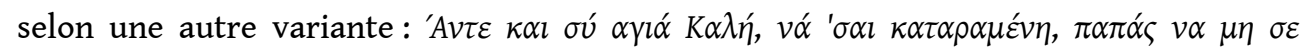

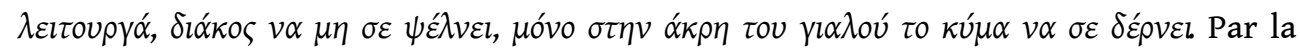
suite, Agia Kalí se repentit et devient une sainte, mais reste maudite et n'est donc jamais fêtée.

27 Agia Kalí est sans doute un personnage mythique lié au cycle des légendes autour de la passion de Jésus Christ. L'exemple le plus célèbre de cette mythologie est le Juif errant, condamné par Jésus Christ à une errance éternelle parce qu'il s'était moqué de lui pendant la montée au mont Golgotha ${ }^{46}$. Agia Kalí pourrait sûrement être la version féminine du Juif. Ce dernier est choisi parce qu'il représente en réalité la communauté juive, premier responsable de la mort du fils de Dieu. Et c'est très possible qu'Agía Kalí ait été choisie pour ce drame de malédiction pour une raison similaire: son origine païenne. Elle est Kalí ton oréon christianisée et finalement sanctifiée.

Les spécialistes $\mathrm{du}$ folklore néohellénique ont souvent soutenu l'affiliationidentification de deux figures. K.A. Romaíos a lié Agia Kalí, d'un côté, à Ino la fille de Cadmos, qui, enragée, s'est précipitée dans la mer et fut métamorphosée par Poséidon en divinité marine sous le nouveau nom de Leucothée, de l'autre, à Kerá Kalí (Kyrá Kaló), l' $\alpha \rho x o ́ v \tau \imath \sigma \sigma \alpha$ (reine) des néraïdes ${ }^{47}$. G. Dimitrokális, auteur de l'article le plus exhaustif sur la figure d'Agía Kalí, a repris cette connexion en la rapprochant aussi à une Kalí marine, yopyóv $\alpha$ (sirène), la sœur d'Alexandre le Grand, originaire de la néréide Kalí. ${ }^{48}$ Par ailleurs, Dimitrokális a tenté de relever tous les problèmes associés à Agía Kalí, comme par exemple l'existence d'un îlot (et d'une Église homonyme) à Naxos qui porte le nom d'Agía Kalí, ou les rapports éventuels du prénom rare Kalí, utilisé déjà depuis le ve siècle av. J.-C., à la nymphe Callisto ou Calliope. Cependant, Kalí a servi de surnom pour plusieurs déesses de l'Antiquité : Aphrodite, Aglaé, la néréide Kalé, Tyché, 
Moira, et une inscription d'Alexandrie atteste le culte même d'une déesse nommée Kalét ${ }^{4}$.

Par conséquent, il devient évident que le nom Kalí a été utilisé pour des déesses de caractère ambivalent ${ }^{50}$ liées à la notion de prospérité. ${ }^{51}$ Agía Kalí ne consiste qu'en la christianisation d'une figure d'origine préchrétienne qui est impliquée dans une fonction prépondérante au sein du paganisme, celle du do ut des, fonction qui a pourtant survécu dans la culture populaire néohellénique. L'imaginaire incluant les

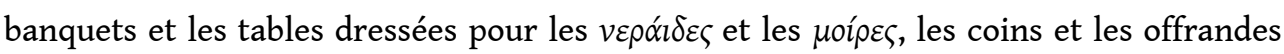

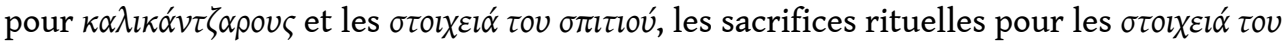

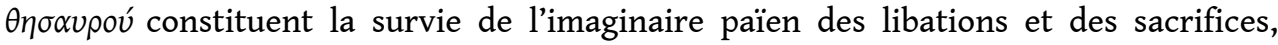
déplacé dans le contexte d'une religion différente, d'une religion qui, par son statut populaire, s'est montrée accueillante, c'est prouvé, pour la cosmologie polythéiste.

\section{BIBLIOGRAPHIE}

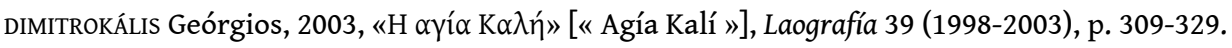

HARF-LANCNER Laurence, 2003, le Monde des fées dans l'occident médiéval, Paris : Hachette.

LAWSON John, 1910, Modern Greek folklore and ancient Greek religion, Cambridge: Cambridge University Press.

LECouteux Claude, 1999, Chasses Fantastiques et Cohortes de la Nuit, Paris : Imago.

MOSER-KARAGIANNIS Emmanuelle, 2005, Littérature orale de la Grèce moderne, Athènes : Dodoni.

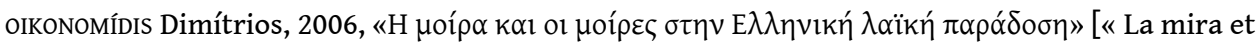
les mires dans la tradition populaire grecque »], Laografía 40, p. 37-79.

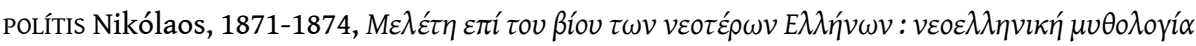
[Étude de la vie des Grecs modernes : mythologie néohellénique], Athènes : A. Pérri, 1871-1874. STEWART Charles, 1991, Demons and the Devil: moral imagination in modern Greek culture, Princeton: Princeton University Press.

\section{NOTES}

1. DELATTE Armand, Anecdota Atheniensia, T. I, Liège : Imp. H. Vaillant-Carmanne ; Paris :

É. Champion, 1927.

2. PUECH Aimé, "Armand Delatte. Anecdota Atheniensia. Tome I. Textes grecs inédits relatifs à l'histoire des religions ", Revue des études grecques, tome 45, fascicule 213, octobre-décembre 1932, p. 459-460.

3. Nous avons choisi pour la traduction de Kalí l'adjectif «bonne » et non pas l'adjectif « belle » pour des raisons qui seront analysées au fur et à mesure de l'article. 
4. Nous soulignons l'importance de cette nourriture qui réapparaîtra dans la majorité des coutumes examinées.

5. Pour une analyse du terme Ephialtès, voir ROSCHER Wilhelm Heinrich, Ephialtes, Eine pathologischmythologische Abhandlung über die Alpträume und Alpdämonen des klassischen Altertums, Leipzig: B. G. Teubner, 1900.

6. Il faut souligner ici qu'Allatios fut le premier à publier le texte mentionné de Psellos. Par la Suite le texte a été publié par l'historien Sáthas. Voir sÁtHAs Konstantínos,

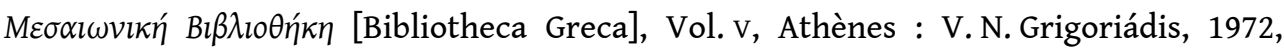
p. 571.

7. AlLATIUS Leo, De templis Graecorum/De Narthece/De Graecorum Hodie, Cologne: Iodocum Kalcovium \& socios, 1645, p. 62.

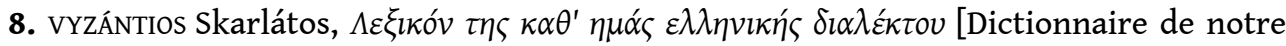
dialecte grecque], Athènes : Tmíma vasilikís typografías, 1835, p. 186.

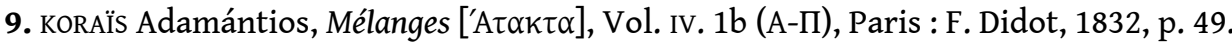

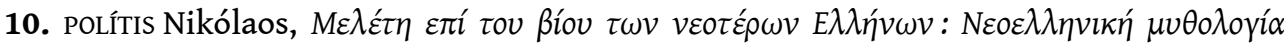
[Étude de la vie des grecs modernes: mythologie néohellénique], Athènes : A. Pérri, 1871-1874, p. 96.

11. Voir Georges DumÉzIL, Mythe et Épopée I (L'Idéologie des trois fonctions dans les épopées des peuples indo-européens), Paris : Gallimard, 1995.

12. Ross Ludwig, Reisen auf den griechischen Inseln des ägäischen Meeres, Band 3, Stuttgart: Tübingen, Gotha, 1845, p. 45.

13. D'ISTRIA Dora, les Femmes en Orient, Vol. I, Zurich : Meyer \& Zeller, 1859, p. 418.

14. DODWELL Edward, Classical and topographical tour through Greece during the years 1801, 1805, and 1806, Vol. I, London: Rodwell and Martin, 1819, pp. 396-397.

15. DODWELL Edward, op. cit., p. 397.

16. AMPÈRE Jean-Jacques, la Grèce, Rome et Dante. Études littéraires, Paris : Didier, p. 60.

17. Pouqueville les appelle ailleurs bonnes (et non pas belles) déesses. Voir POUQUEVILLE François, Voyage dans la Grèce, Vol. v, Paris : Firmin Didot, 1827, p. 577.

18. La relation entre mires (parques) et néraïdes (fées) est évidente, non seulement dans le monde néohellénique comme Pouqueville le relève. En français, par exemple, le nom fée dérive du latin fata, ensuite de fatum (du verbe for, parler), "celui qui est dit », le Destin. Voir HARF-LANCNER Laurence, le Monde des fées dans l'occident médiéval, Paris : Hachette, 2003, p. 17-18.

19. POUQUEVILLE François, Voyage dans la Grèce, Vol. Iv, Paris : Firmin Didot, 1820, p. 419.

20. POUQUEVILLE François, Voyage dans la Grèce, Vol. v, Paris : Firmin Didot, 1827, p. 64.

21. Ibid., p. 67.

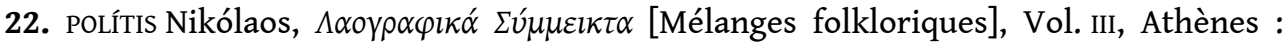
Académie d'Athènes, 1931, p. 200.

23. Archive du Centre de recherches du folklore hellénique (Académie d'Athènes) : $n^{\circ} 1378$ ŕ, p. 164.

24. Ibid., $\mathrm{n}^{\circ}$ 7, p. 216.

25. Ibid., nº 180 , p. $149,16$. 


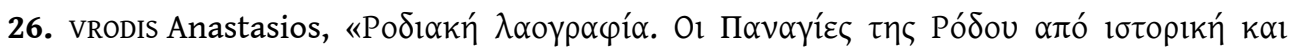

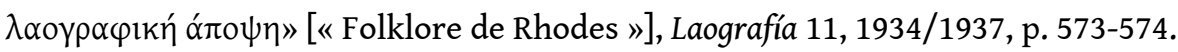

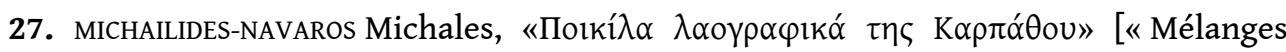
folkloriques de Karpathos »], Laografía 16, 1956, p. 115.

28. Ed. MEINEKE August, Aristophanes Comoediae, Vol. I, Leipzig: Tauchnitz, 1860, p. 123,

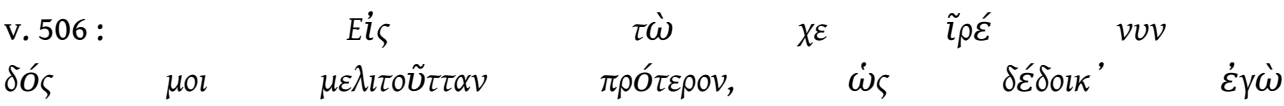

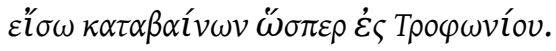

29. Trad. CLAVIER Étienne, Description de la Grèce de Pausanias, Vol. v, Paris : Société Royale Académique des Sciences, 1821, p. 232.

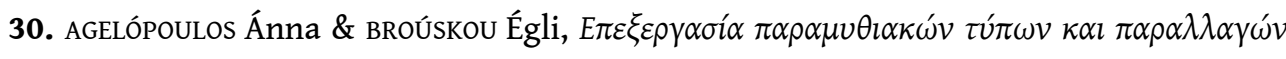
AT 300-499 [Traitement des types des contes et des variantes AT 300-499], Vol. I, Athènes : E.I.E., 1999, p. 645.

31. Des variantes du type AT 410 ont été trouvées en Thrace, aux îles égéennes, aux îles ioniennes, au Péloponnèse, en Grèce centrale. Pour plus d'informations, voir: AGELóPoulos Ánna \& BRoúsKou Égli, op.cit., p. 645-651.

32. Le Roman de Perceforest, Troisième partie, T. III, éd. Gilles Roussineau, Genève : Droz, 1993.

33. Césaire d'Arles ( $\mathrm{VI}^{\mathrm{e}}$ siècle), saint Boniface ( $\mathrm{VII}^{\mathrm{e}}-\mathrm{VIII}^{\mathrm{e}}$ siècles), le pseudo-Augustin (vIII ${ }^{\mathrm{e}}$ siècle), le pseudo-Eloi de Noyon (IX siècle), Atto de Vercelli ( $\mathrm{x}^{\mathrm{e}}$ siècle), Yves de Chartres ( $\mathrm{xI}^{\mathrm{e}}$ siècle), Gratien (xII ${ }^{\mathrm{e}}$ siècle), Guillaume d'Auvergne (XII ${ }^{e}$-XIII ${ }^{e}$ siècles), le pape Jean XXI ( $\mathrm{XV}^{\mathrm{e}}$ siècle).

34. MOSER-KARAGIANNIS Emmanuelle, Littérature orale de la Grèce moderne, Athènes : Dodóni, 2005, p. 283.

35. Ibid., p. 283.

36. Ibid.

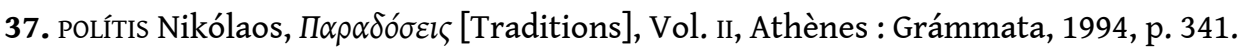

38. Pour plus d'informations sur le genius loci néohellénique, voir: PoLítis Nikólaos,

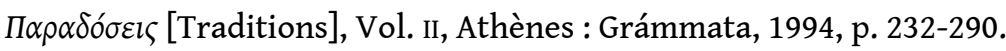

39. ALLATIUS Leo, op. cit., p. 163-168.

40. D’après la théorie de Mircea Eliade. Voir : ELIADE Mircea, le Sacré et le Profane, Paris : Gallimard, 1965.

41. Pour plus d'informations sur le génie des trésors en Grèce moderne, voir zocHIós Stamátis, "Treasure Spirits and Guardians: between dream and illusion", Caietele Echinox, Vol. 23, Roumanie : Cluj-Napoca 2013, pp. 59-68.

42. Qui est indéniablement chrétien, pourrait-on ajouter. Pour une analyse profonde de la cosmologie grecque, voir : STEWART Charles, Demons and the Devil: moral imagination in modern Greek culture, Princeton: Princeton University Press, 1991.

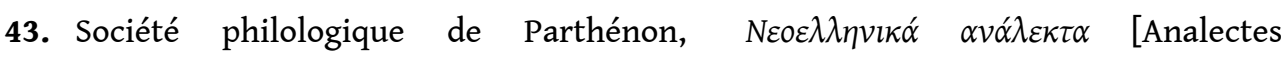
néohelléniques], Vol. I, Athènes : Súllogos Parthenón, 1870, p. 335.

44. Motif relevant de la relation des néraïdes ou des fées, avec l'eau. 


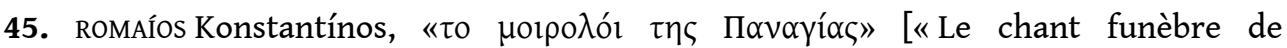
Panagía »], Archeíon Póntou, Vol. xIX, 1954, p. 98.

46. Pour plus d'informations sur la mythologie du Juif errant, voir: MILIN Gaël, le Cordonnier de Jérusalem. La Véritable Histoire du Juif errant, PUR, 1997.

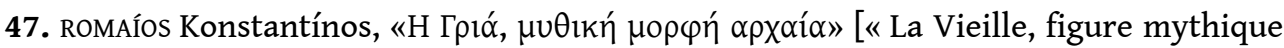
ancienne »], Prosforá eis Stílpona Kiriakídi, Thessalonique, 1953, 574.

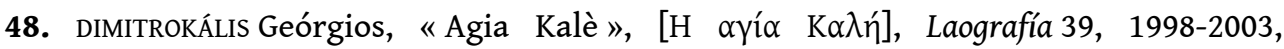
p. 316-319.

49. Ibid.

50. L'aspect maléfique de Kerá Kalí est documenté dans une incantation crétoise contre

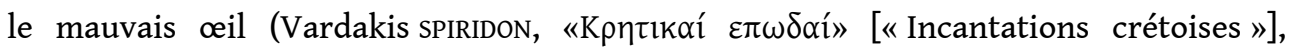
Laografia 10, 1926/1928, p. 245-246). Celle-ci raconte que Kerá Kalí est partie ayant les cheveux lâchés - trait des figures mythologiques démoniaques - pour faire du mal aux

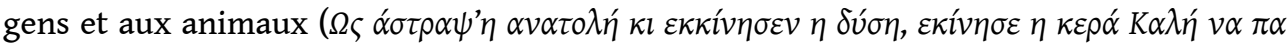

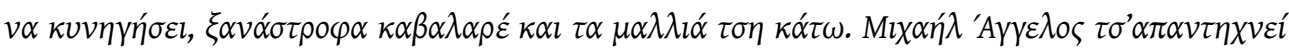

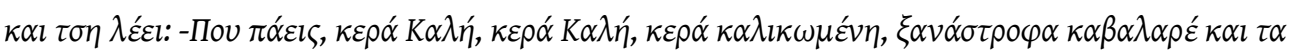

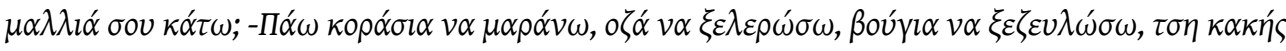

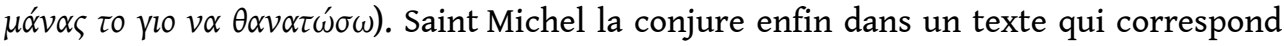
aux fameux exorcismes gialloudochartia. Kerá Kalí remplace dans ce cas le démon féminin Gylou (Gylo ou Gelo). Pour plus d'informations sur les papiers de Gylou et ses historiolae, voir : PATERA Maria, «Exorcismes et phylactères byzantins : écrire, énoncer les noms du démon", Cahiers "Mondes anciens » [En ligne], 1|2010, mis en ligne le 20 janvier 2010, consulté le 6 septembre 2015. URL: http://mondesanciens.revues.org/ 139, DOI : 10.4000/mondesanciens.139.

51. Cette coutume ne concerne pas uniquement la Grèce. Bona Dea, par exemple, est une déesse romaine de fertilité et de prospérité, souvent liée à des banquets et tables dressées tout comme les kalés kuries et les bonnes dames de la mythologie française.

\section{RÉSUMÉS}

Dans les récits populaires néo-helléniques, le peuple peut obtenir la faveur de certaines figures surnaturelles féminines, souvent appelées Kalés Kuries, néraïdes et mires. Pour les accueillir, il lui fallait préparer la table d'une manière particulière, en disposant dessus un certain type de nourriture, différentes sortes de boissons, ainsi que des couverts. Si les dames étaient satisfaites des offrandes, elles donnaient à l'hôte des cadeaux - matériels ou non - pour le récompenser.

L'objectif de cet article est d'étudier les différentes manières dont la nourriture est utilisée par l'homme pour établir un lien avec le surnaturel, dans les croyances populaires néo-helléniques concernant les êtres fantastiques.

In Modern Greek folk tales, men can obtain favour of some female supernatural figures, often called Kales Kuries, neraides or mires. To accommodate them, people had to prepare the table in a particular way, by displaying a certain type of food, different kinds of drinks and cutlery. If the 
women were satisfied with the offerings, they were rewarding the person with material or immaterial gifts.

The aim of this article is to cast light on the ways in which people use food in order to get in contact with the supernatural, in Modern Greek popular beliefs about imaginary beings.

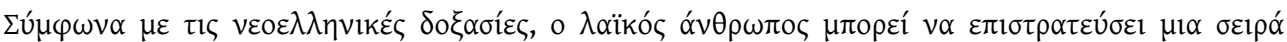

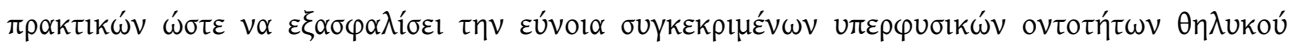

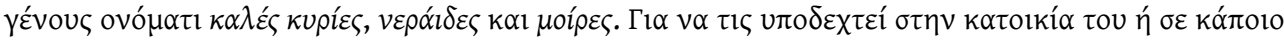

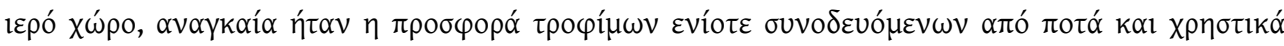

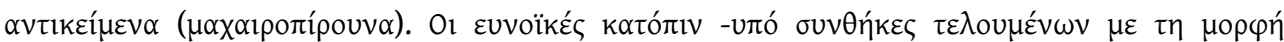

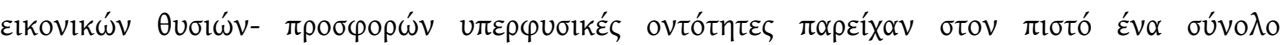

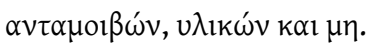

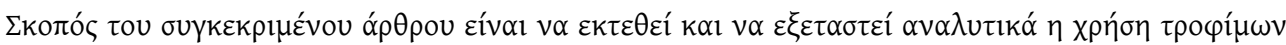

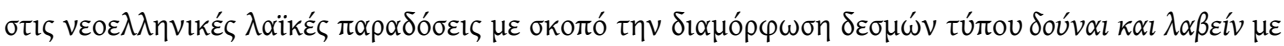

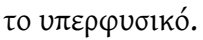

\section{INDEX}

Index géographique : Grèce

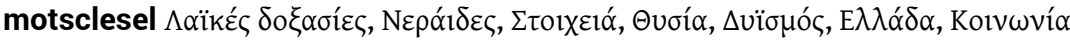
motsclestr Popüler İnançlar, Periler, Kurban, Düalizm, Yunanistan, Toplum motsclesmk ПОПУЛАРНИ ВЕРУВАњА, САМОВИЛИ, ЖРТВА, ДУАЛИЗМОТ, ГРЦИЈА, СОЦИЈАЛНАТА

Keywords : Folk beliefs, Fairies, Sacrifice, Dualism, Greece, Society

Mots-clés : croyances populaires, génies, sacrifices, dualisme

\section{AUTEUR}

\section{STAMATIS ZOCHIOS}

Université de Strasbourg 